3

4

\title{
Soybean Haplotype Map (GmHapMap): A Universal Resource for Soybean Translational and Functional Genomics
}

Davoud Torkamaneh ${ }^{1,2,3}$, Jérôme Laroche ${ }^{2}$, Babu Valliyodan ${ }^{4}$, Louise O’Donoughue ${ }^{5}$, Elroy Cober $^{6}$, Istvan Rajcan ${ }^{3}$, Ricardo Vilela Abdelnoor ${ }^{7,8}$, Avinash Sreedasyam ${ }^{9}$, Jeremy Schmutz ${ }^{9,10}$, Henry T. Nguyen ${ }^{4}$, François Belzile ${ }^{1,2^{*}}$

${ }^{1}$ Département de Phytologie, Université Laval, Québec City, QC, Canada

${ }^{2}$ Institut de Biologie Intégrative et des Systèmes (IBIS), Université Laval, Québec City, QC, Canada

${ }^{3}$ Department of Plant Agriculture, University of Guelph, Guelph, ON, Canada

${ }^{4}$ National Center for Soybean Biotechnology and Division of Plant Sciences, University of Missouri, Columbia, MO, USA

${ }^{5}$ CÉROM, Centre de recherche sur les grains inc., Saint-Mathieu de Beloeil, QC, Canada

${ }^{6}$ Agriculture and Agri-Food Canada, Ottawa, ON, Canada

${ }^{7}$ Brazilian Corporation of Agricultural Research (Embrapa Soja), Carlos João Strass road, Warta County, PR Brazil

${ }^{8}$ Londrina State University (UEL), Londrina, PR, Brazil

${ }^{9}$ HudsonAlpha, Institute for Biotechnology, Huntsville, AL, USA

${ }^{10}$ Department of Energy, Joint Genome Institute, Walnut Creek, CA, USA 


\section{Abstract}

2 Here we describe the first worldwide haplotype map for soybean (GmHapMap) constructed using

3 whole-genome sequence data for 1,007 Glycine max accessions and yielding 15 million variants.

4 The number of unique haplotypes plateaued within this collection (4.3 million tag SNPs)

5 suggesting extensive coverage of diversity within the cultivated germplasm. We imputed

6 GmHapMap variants onto 21,618 previously genotyped (50K array/210K GBS) accessions with

7 up to $96 \%$ success for common alleles. A GWAS performed with imputed data enabled us to

8 identify a causal SNP residing in the NPC1 gene and to demonstrate its role in controlling seed

9 oil content. We identified 405,101 haplotypes for the 55,589 genes and show that such haplotypes

10 can help define alleles. Finally, we predicted 18,031 putative loss-of-function (LOF) mutations in

11 10,662 genes and illustrate how such a resource can be used to explore gene function. The

12 GmHapMap provides a unique worldwide resource for soybean genomics and breeding. 


\section{Introduction}

2 Soybean (Glycine max [L.] Merr.) is a unique crop with substantial economic value. It is the

3 largest plant source of both animal feed protein and edible oil. It also plays a key role in

4 sustainable agriculture as it fixes atmospheric nitrogen with the help of microorganisms

5 (Hymowitz 1970). Diverse evolutionary processes and forces (including cycles of polyploidization

6 and subsequent diploidization), along with domestication and modern breeding have shaped the

7 soybean genome (Schmutz et al. 2010). The detection of the molecular footprints of these processes

8 is essential for understanding how genetic diversity is generated and maintained and for identifying

9 allelic variants responsible for phenotypic variation (Torkamaneh et al. 2018).

10 The global production of soybean has increased substantially in recent years (Supplementary

11 Figure 1), but the rate of annual yield gains has lagged behind that of maize (FAOSTAT

12 Database). In addition, with increased fluctuations in climatic conditions, next-generation soybean

13 cultivars must not only be higher yielding but also more resilient to multiple abiotic and biotic

14 stresses (Djanaguiraman et al. 2018). In the main soybean-growing areas of the world, soybean is

15 an introduced crop and the foundational germplasm was very limited in its genetic diversity (Hyten

16 et al. 2006; Maldonado dos Santos 2016). Continued genetic improvement in soybeans will require

17 a better understanding of the genetic and especially allelic diversity within worldwide resources

18 (Qiu et al. 2013).

19 Here we present the first haplotype map for soybean (GmHapMap) assembled from DNA

20 resequencing data for a collection of 1,007 worldwide G. max accessions. We explore the use

21 of this GmHapMap for (i) imputation of untyped variants to create high density genotype data

22 required for gene-level resolution of genomewide association studies (GWAS); (ii) construction

23 of gene-centric haplotypes (GCHs) for the entire set of soybean genes; and (iii) identification 
1 of $11 \mathrm{~K}$ knock-out genes due to loss-of-function (LOF) mutations. The GmHapMap provides a

2 unique resource for translational and functional genomics for the worldwide soybean community.

4 Results

\section{Development of GmHapMap}

\section{Genomic variation}

7 To establish a first worldwide haplotype map for soybean (GmHapMap), a total of 1,007

8 resequenced soybean accessions, representative of the worldwide cultivated germplasm, were used

9 (Figure 1A). These accessions span thirteen maturity groups (MGs) (000-X) based on their

10 latitudinal adaptation. This collection includes 727 previously resequenced accessions, as well as

11280 accessions sequenced as part of this study which were selected to achieve a more complete

12 coverage of soybean worldwide diversity. Genome sequencing, analyses, and accession

13 information for GmHapMap accessions are summarized in a Supplementary Note and

14 Supplementary data 1.

15 In total, 165 billion paired-end reads (100-150 bp; total of 19.2 trillion bp) provided an average

16 depth of coverage of more than $15 \times$ and these were analyzed using a single pipeline (Fast-WGS)

17 to ensure uniform variant calling. After mapping against the soybean reference genome (cv.

18 Williams 82 va2.v1) (Schmutz et al. 2010), we identified 14,872,592 nucleotide variants (Table

19 1), including $13 \mathrm{M}$ single- and multiple-nucleotide variants (SNVs and MNVs) and $2 \mathrm{M}$ small

20 insertions/deletions (InDels) ( $-50 \mathrm{bp}$ to $+32 \mathrm{bp}$ ). Approximately $45 \%$ of these were rare (minor

21 allele frequency $(\mathrm{MAF})<5 \%$ ) (Supplementary Figure 2). Coding regions represent $\sim 6 \%$ of the

22 soybean genome, but only $\sim 2.3 \%$ of the total nucleotide variants were present in these regions 
1 (Table 1) with an average non-synonymous/synonymous ratio of 1.49 . Nucleotide variants were

2 2-fold more abundant in coding regions compared to InDels, however InDels were overrepresented

3 in the regulatory regions. Missing data comprised less than $8 \%$ of the data, and these were

4 subsequently imputed with high accuracy $\left(r^{2}=99.7 \%\right)$. Using independent genotyping data (SNP

5 array and dbSNP database), we estimated the false-positive rates of nucleotide variants to be

$6 \quad 0.03 \%$. This constitutes an extensive and highly accurate set of foundational data for a soybean

7 haplotype map.

8

9 Table 1. Type, number and location of nucleotide variants in GmHapMap.

\begin{tabular}{lr|rrrrrr}
\hline \multicolumn{2}{c|}{ Statistics } & \multicolumn{6}{c}{ Location (\%) } \\
\hline Types & Count & CDS* & Intron & UTR $\uparrow$ & $\begin{array}{c}\text { Splice } \\
\text { sites }\end{array}$ & $\begin{array}{c}\text { Up/Down } \\
\text { stream }+\end{array}$ & Intergenic \\
\hline SNV & $12,197,920$ & 2.5 & 8.5 & 1.6 & 0.2 & 44.8 & 42.4 \\
MNV & 801,373 & 2.3 & 6.9 & 1.0 & 0.1 & 44.6 & 45.1 \\
INS & 887,485 & 0.9 & 10.2 & 2.7 & 0.2 & 54.3 & 31.6 \\
DEL & 985,814 & 1.0 & 10.1 & 2.3 & 0.3 & 53.0 & 33.2 \\
\hline Total & $\mathbf{1 4 , 8 7 2 , 5 9 2}$ & $\mathbf{2 . 8}$ & $\mathbf{8 . 6}$ & $\mathbf{1 . 7}$ & $\mathbf{0 . 2}$ & $\mathbf{4 5 . 9}$ & $\mathbf{4 1 . 7}$ \\
\hline
\end{tabular}

10

11

12

13

14

15

16

17 rare SNPs $(\mathrm{MAF}<1 \%$ ) documented within these $G$. $\max$ accessions. Haplotype diversity 
1 (pairwise LD using both $\mathrm{r}^{2}$ and $\mathrm{D}^{\prime}$ ) was calculated for sequence variants and the average distance

2 over which LD decayed to 0.2 was $\sim 138 \mathrm{~kb}$ (Supplementary Figure 3). We identified 4.3 million

3 haplotype-based tag SNPs and, to determine if a good level of saturation of both variants and

4 haplotypes had been achieved, we randomly selected subsets of samples of increasing size $(\mathrm{N}=100$,

$5200, \ldots$, and 1,007). As illustrated in Figure 1B, the number of variants discovered did not increase

6 significantly beyond $\sim 750$ accessions, while the number of haplotypes reached a plateau much

7 faster (within the first $\sim 500-600$ accessions). Together, these results suggest that the GmHapMap

8 dataset offers an exhaustive characterization of the variants and haplotypes present in soybean

9 germplasm.

\section{Genetic diversity and artificial selection}

12 Bayesian clustering (STRUCTURE) of GmHapMap accessions using whole-genome SNP data

13 revealed 12 subpopulations (Supplementary Note \& Supplementary Figure 4). We explored the

14 phylogenetic relationships among GmHapMap accessions by constructing an un-rooted neighbor-

15 joining tree. As can be seen in Figure 1C, the grouping of accessions reflected geographic origin

16 with some admixture (Figure 1C and Supplementary Figure 5). Genomewide genetic diversity

$17\left(\theta_{\pi}\right)$ analysis showed a consistent level of genetic diversity (mean of $\theta_{\pi}=1.36 \times 10^{-3}$, ranging

18 between $1.19 \times 10^{-3}$ to $1.72 \times 10^{-3}$ ) in different soybean populations (Figure $\left.\mathbf{1 A}\right)$. Nucleotide

19 diversity was plotted for the 20 chromosomes and found to be highest in the terminal regions of

20 chromosomes (Supplementary Figure 6). Extensive peri-centromeric regions were very low in

21 genetic diversity but chromosomes Chr13 and Chr17 maintained higher diversity across these

22 regions. Genic regions showed even lower levels of diversity (mean $\theta_{\pi}=7.1 \times 10^{-4}$ ) and

23 exceptionally low diversity was seen within 527 genomic regions (including 540 genes; mean $\theta_{\pi}$ 
bioRxiv preprint doi: https://doi.org/10.1101/534578; this version posted January 29, 2019. The copyright holder for this preprint (which was not certified by peer review) is the author/funder, who has granted bioRxiv a license to display the preprint in perpetuity. It is made available under aCC-BY 4.0 International license.

$1=4.6 \times 10^{-6}$ ) that are presumably selection hotspots (Supplementary Note, Supplementary data

\section{$2 \quad 2$ and Supplementary Figure 7).}

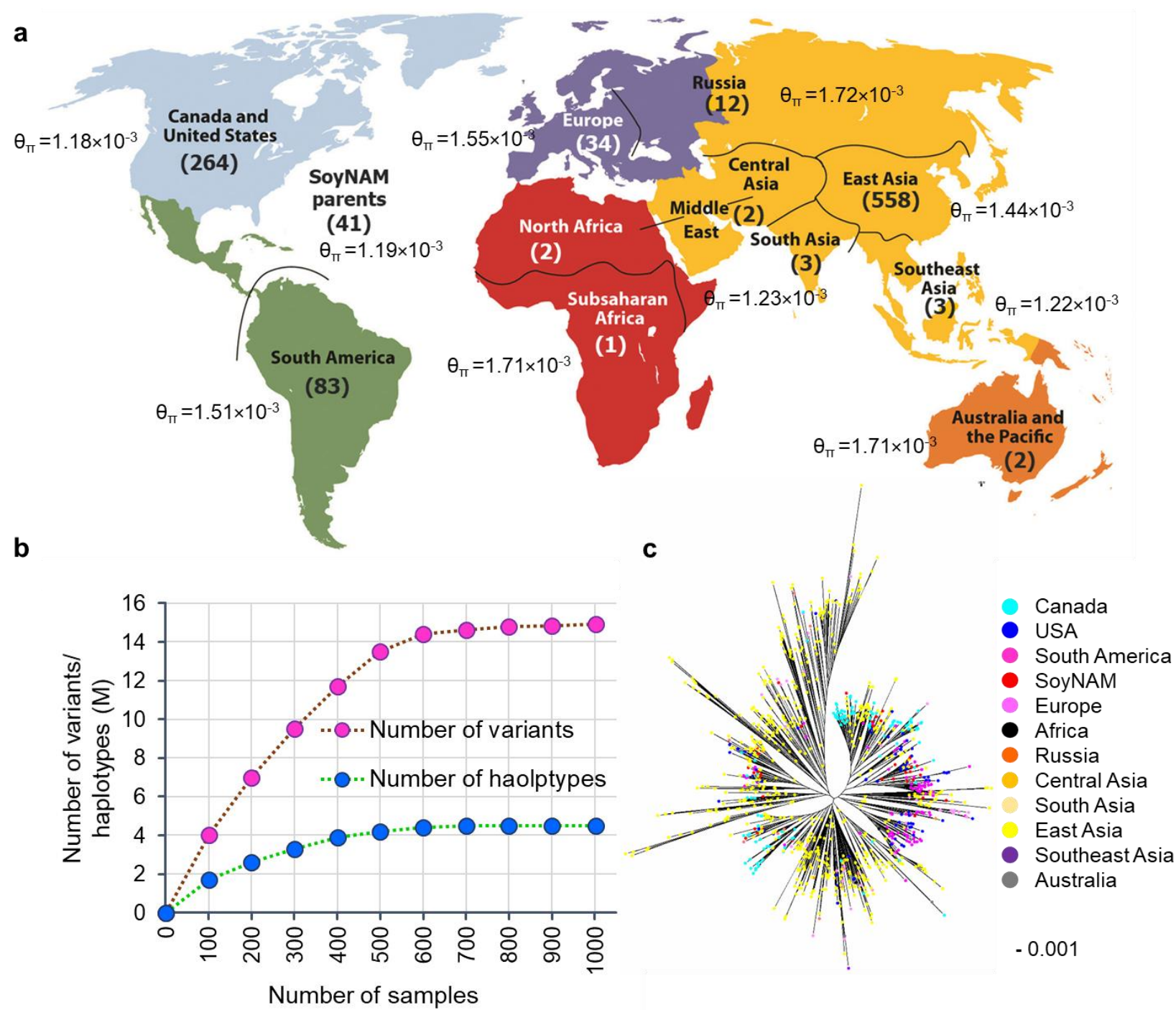

Figure 1. Description of GmHapMap. (a) Geographical distribution and related genetic diversity value $\left(\theta_{\pi}\right)$ of GmHapMap accessions. (b) Number of variants (pink) and haplotypes (blue) based on different number of accessions. (c) Un-rooted phylogenetic tree of all accessions inferred from

7 whole-genome SNPs representing existing genetic diversity and admixture among GmHapMap accessions. 


\section{Phasing, identity-by-descent, and large-scale imputation of untyped variants}

2 The GmHapMap dataset captures substantial amounts of identity-by-descent (IBD) allele sharing which allows a rule-based approach to long-range phasing that yields very accurate haplotypes.

4 Using long-range phasing, we found 95 blocks of IBD larger than $1 \mathrm{Mb}$ in size (Supplementary data 3). The determination of haplotype phase is important because of its applications such as the imputation of untyped variants. Imputation of untyped variants greatly boosts variant density,

7 allowing fine-mapping studies of GWAS loci and large-scale meta-analysis. We created two reference panels: REF-I comprising all SNPs and REF-II containing 1.9M haplotype-based tag SNPs that reside in genic regions. Three lower density genotype datasets, SoySNP50K (20,087 accessions genotyped with 43K SNPs), genotyping-by-sequencing (GBS; 1,531 accessions genotyped with 210K SNPs), and combined GBS/SoySNP50K (1,531 accessions genotyped with 250K SNPs) were used for untyped variant imputation with each of the two reference panels. In all but one case, the accuracy (squared correlation $\left(R^{2}\right)$ between imputed and known genotypes, see M\&M for details) ranged between $92 \%$ and $96 \%$ for common variants (allele frequency (AF) $>0.2$ ) in each dataset, while decreasing gradually with allele frequency (Figure 2A). In the case of the SoySNP50K dataset using REF-I, the accuracy of imputed untyped variants was significantly lower (80-85\% for common alleles). Given the observed variation in the accuracy of imputation using different reference panels and datasets, we investigated the causes of erroneous inferred calls. Several characteristics were tied to inaccurately imputed SNPs: these were commonly rare variants (low AF), located in recombination hot spots, in short LD blocks or in

21 genomic regions with structural variants. Furthermore, the initial marker density in the 22 experimentally-derived dataset had a large impact on imputation accuracy. GBS and SNP array 23 datasets are two highly complementary marker datasets because most ( $90 \%)$ of the SoySNP50K 
1 markers are present in genic regions, while most of the GBS markers $(\sim 60 \%)$ are present in

2 intergenic regions (Supplementary Figure 8 \& 9). Therefore, combining GBS and SoySNP50K

3 datasets (Supplementary Note) increases the density and uniformity of distribution of SNPs

4 across the genome. The joint use of such commonly available SNP data increased the level of

5 accuracy of imputation of untyped variants (Figure 2A).

6 To demonstrate the benefits of untyped-variant imputation on GWAS analysis, the imputation was

7 performed on a $1 \mathrm{Mb}$-region harbouring a QTL previously identified for seed oil content on

8 chromosome 14. We used the REF-II panel to perform imputation on an initial dataset of $64 \mathrm{~K}$

9 GBS-derived SNPs (genomewide) among 139 soybean lines that had been characterized for their

10 seed oil content (Sonah et al. 2015). Using this enhanced SNP catalog and a multi-locus mixed-

11 model implementation, a very strong association ( $p$-value $=4.2 \times 10^{-14}$ and $q$-value $\left.<0.001\right)$ with a

12 SNP residing in the NPC1 (Niemann-Pick C1) gene (Glyma.14g001500) (Figure 2B) was

13 detected. An Arabidopsis mutant of this gene (npcl) exhibits a 58\% higher fatty acid content

14 (Feldman et al. 2015) making this gene a likely candidate contributing to total oil content in

15 soybean. This demonstrates that the increased number of informative SNP loci, obtained through

16 the imputation of untyped variants, can prove highly beneficial in studying the genetic architecture

17 of complex agronomic traits in soybean. 
bioRxiv preprint doi: https://doi. org/10.1101/534578. this version posted January 292019 . The copyright holder for this preprint (which was not certified by peer review) is the author/funder, who has granted bioRxiv a license to display the preprint in perpetuity. It is made available under aCC-BY 4.0 International license.

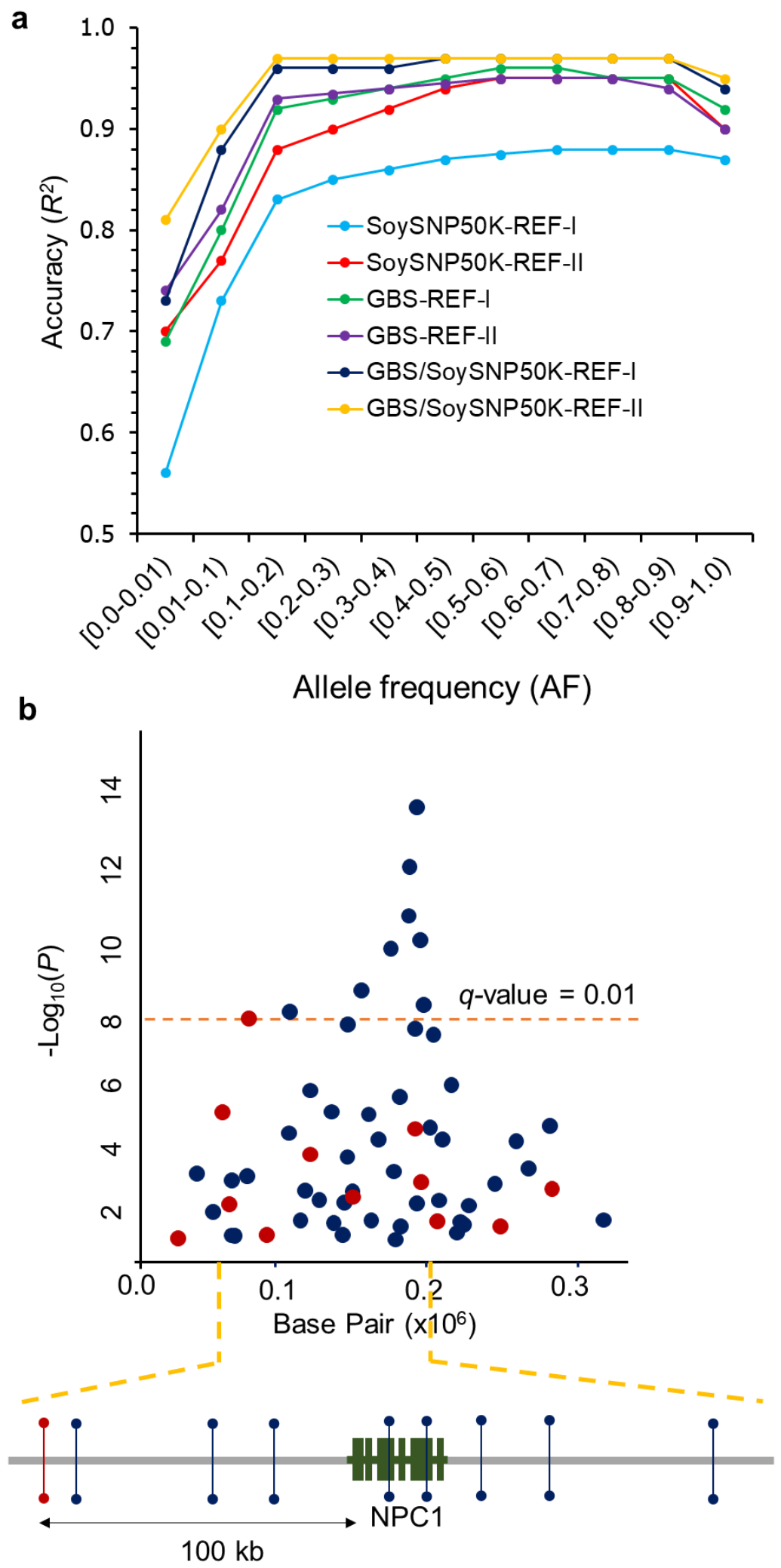


1 Figure 2. (a) Imputation accuracy as a function of allele frequency for 6 different scenarios; three

2 different experimentally derived genotype datasets (SoySNP50K, GBS, and GBS/SoySNP50K)

3 and two reference panels (REF-I and REF-II). (b) Top, association analysis for seed oil content on

4 chromosome 14. Blue dots represent imputed variants whereas red dots identify the original GBS-

5 derived variants. NPCl (Niemann-Pick C1) is an orthologue of an Arabidopsis gene known to play

6 a key role in fatty acid synthesis. Bottom, schematic representation of strongly associated variants

7 in the vicinity of the NPC1. The nearest significantly associated GBS-derived variant (red line) is

8 located $100 \mathrm{~kb}$ upstream and exhibits a relatively low degree of $\mathrm{LD}\left(\mathrm{r}^{2}=0.5\right)$.

11 Gene-centric haplotypes: a resource for translational genomics

12 HaplotypeMiner (Tardivel et al. unpublished) and the GmHapMap SNP dataset were used to identify 405,101 gene-centric haplotypes (GCHs) for 52,823 genes (94.5\% of all soybean genes

$14(55,589))$. As can be seen in Figure 3A, the number of GCHs per gene ranged between 2 and 43, while averaging $\sim$ (Supplementary Figure 10, and Supplementary data 4). GCHs could not be determined (ND) for 2,766 genes with the set of parameters used here. In total, 11,407 genes

17 had more than $10 \mathrm{GCHs}$ with $71 \%$ (8,082 genes) of these harboring 11-15 GCHs. Such genes were typically located in very short LD blocks with a high degree of nucleotide diversity $\left(\right.$ mean $\theta_{\pi}=4.5$

$\left.19 \times 10^{-3}\right)($ Supplementary Figure 11). A slight negative correlation was observed between gene length and the number of GCHs. However, we found a positive correlation between GCH counts 21 and haplotype size (distance between two most distant SNPs defining a GCH) (Supplementary 22 Figure 12). An example of GCHs for the GmGIa (Glyma.10g221500) gene (E2 locus controlling 
1 maturity) (Watanabe et al. 2012; Tsubokura et al. 2014), an orthologue of the arabidopsis

2 GIGANTEA $(G I)$ gene, is presented in Figure 3B. We found three GCHs for GmGIa, which is

3 consistent with the number of alleles that have been previously reported for this gene. Knowledge

4 of the GCHs (and possibly alleles) in all soybean genes can greatly facilitate the establishment of

5 a functional link between the various alleles of a gene and the associated phenotype.

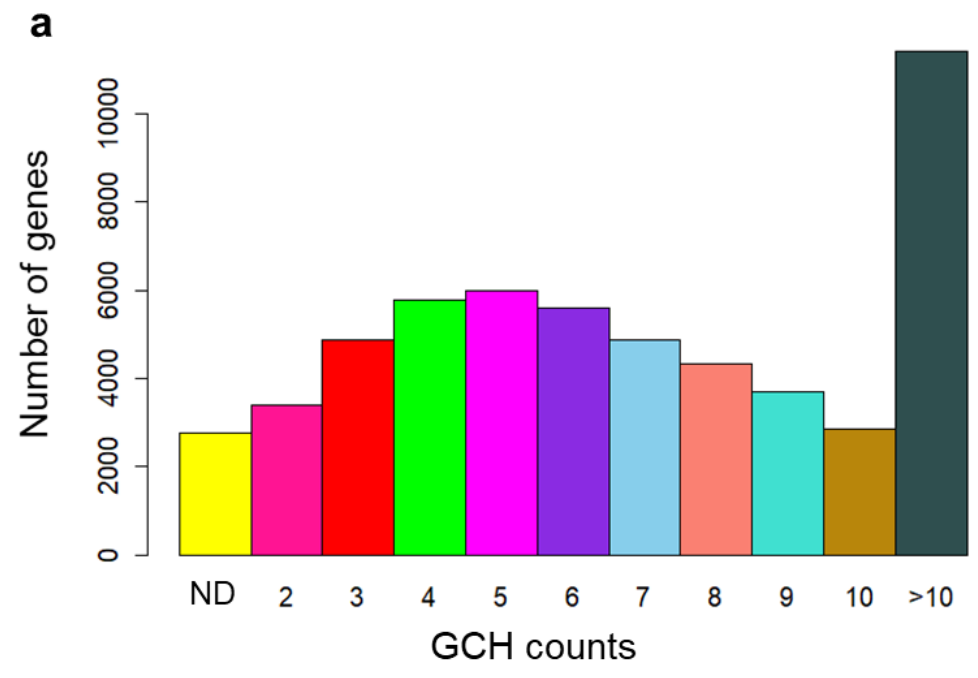

b

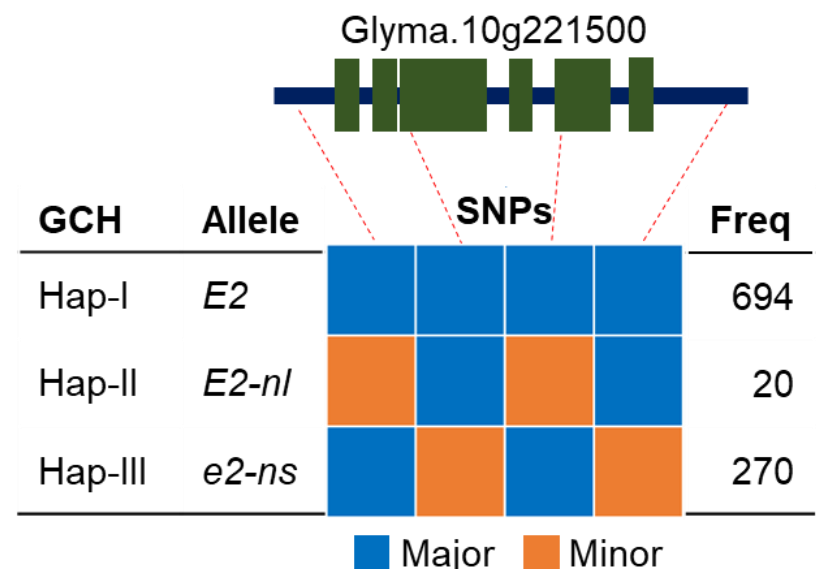

7 Figure 3. Description of GCHs characterized in GmHapMap dataset. (a) Distribution of number

8 of genes based on their predicted GCHs. (b) Schematic representation of predicted GCHs for

9 GmGIa. 


\section{LOF Mutations: a resource for functional genomics}

2 Using SnpEff, a subset of variants located inside the coding regions were predicted to have a large

3 functional impact. Of these variants, 18,031 putative loss-of-function (LOF) mutations are

4 predicted to severely impair protein synthesis or function through disruption of splicing,

5 introduction of a premature stop codon, shifts in the coding frame and alterations to the start/stop

6 codons (MacArthur et al. 2012) and these were identified in a total of 10,662 genes (19.3\% of all

7 soybean genes) (Table 2). These mutations are the result of 5,987 SNVs (33.2\%), 279 MNVs

$8 \quad(1.5 \%)$ and 11,765 InDels (65.3\%). Frameshift-inducing variants $(10,754)$ were the predominant

9 category, representing 59.6\% of LOF mutations and affecting 6,718 genes. InDels (ranging from

$10-50 \mathrm{bp}$ to $+32 \mathrm{bp}$ ) were, understandably, over-represented (4-fold) in the LOF category due to their

11 high probability of resulting in a LOF allele. Overall, most of the LOF mutations were present at

12 low frequency, with 78\% having an allele frequency below 10\% (Supplementary Figure 13).

13 Genes harboring LOF one or more mutations were categorized into two groups: unique and multi-

14 copy. We reasoned that a LOF mutation in a unique gene would necessarily result in phenotypic consequences. We found that only $706(6.6 \%)$ of genes were single-copy genes, while the remaining 9,957 (93.4\%) had at least one other copy. This constitutes a significant enrichment $(P$

$17<0.001)$ compared to the genomewide occurrence of gene duplication. LOF mutations in duplicated genes could also have functional consequences if the mutated copy was uniquely

19 expressed as a consequence of neo- or sub-functionalization (Roulin et al. 2013). We assessed this

20 by examining transcriptomic data from 26 tissues and found that 9,570 of the 9,957 duplicated

21 genes (96\%) exhibited a unique expression pattern (Supplementary Note, Supplementary data

$225 \&$ Supplementary Figure 14). Thus, despite the fact that the vast majority of LOF mutations

23 occur in genes for which there is more than one copy, a large proportion of these genes exhibit 
1 unique expression patterns, thus making it possible that a LOF will result in a detectable

2 phenotype.

4 Table 2. Number of loss-of-function variants by sequence ontology (SO).

\begin{tabular}{lrrrrrr}
\hline SO term & SNV & MNV & INS & DEL & $\begin{array}{c}\text { Total } \\
\text { variants }\end{array}$ & Genes \\
\hline Splice site-disrupting (donor) & 1,270 & 38 & 247 & 205 & 1,760 & 1,640 \\
Splice site-disrupting (acceptor) & 1,546 & 52 & 207 & 146 & 1,951 & 1,803 \\
Stop codon-introducing & 2,826 & 149 & 100 & 7 & 3,082 & 2,418 \\
Frameshift-inducing & 0 & 0 & 4,158 & 6,596 & 10,754 & 6,718 \\
Start/Stop codon-disrupting & 345 & 40 & 54 & 45 & 484 & 452 \\
Total & $\mathbf{5 , 9 8 7}$ & $\mathbf{2 7 9}$ & $\mathbf{4 , 7 6 6}$ & $\mathbf{6 , 9 9 9}$ & $\mathbf{1 8 , 0 3 1}$ & $\mathbf{1 3 , 0 3 1}$ \\
\hline Total number of genes affected by LOF variants* & & & & & $\mathbf{1 0 , 6 6 2}$ \\
\hline
\end{tabular}

* Some of the genes were affected with more than one LOF mutation, therefore the total number

6 of genes is lower than the sum of the all genes.

To assess the quality of this catalogue of mutations, we first inspected it for genes already known

9 (i.e. functionally validated) to harbor an LOF mutation. This is indeed the case, all known genes in the literature were found within the catalogue (Supplementary data 6). Then we investigated

11 and confirmed the phenotypic impact of some of these LOF mutations in GmHapMap accessions

12 (Figure 4). A frameshift mutation (frequency=0.003) in the microsomal omega-3 fatty acid 13 desaturase $(F A D 3 A)$, a key gene for linolenic acid synthesis in soybean seeds (Reinprecht \& Pauls 14 2016), was found in three accessions. Near-infrared spectroscopy (NIRS) analysis of four soybean 15 lines (two with and without this LOF mutation) showed a significant $(P<0.01)$ decrease in 16 linolenic acid content in the mutant lines (4\%) compared to the wild type (10\%) (Figure 4A). A 17 mutation $(\mathrm{f}=0.005)$ in Glyma.04G050200, the gene underlying the $\mathrm{J}$ locus controlling the Long 18 Juvenile trait (Lu et al. 2017), resulted in a significant difference $(P<0.01)$ in grain weight per 
1 plant ( $8 \mathrm{~g}$ in the mutant compared to $25 \mathrm{~g}$ in the wild type) (Figure 4B). The introduction of a

2 premature stop codon ( $\mathrm{f}=0.02)$ due to a SNV in GmGIa/E2 (Watanabe et al. 2012) significantly $(P$

$3<0.01)$ reduced the number of days from emergence to the appearance of the first open flower

4 (DAE) (from 125 in wild-type lines to 95 in the mutant) (Figure 4C). Finally, a SNV (f=0.009)

5 resulted in the disruption of splicing in the gene coding for the 3-ketoacyl-ACP synthase II (KASII)

6 enzyme, a key gene in the oil biosynthesis pathway (Goettel et al. 2016). NIRS analysis of palmitic

7 acid levels showed a significant $(P<0.05)$ decrease in the mutant lines $(9 \%)$ compared to the wild

8 type (12\%) (Figure 4D). The development of a catalogue of LOF mutations represents a valuable

9 resource for functional genomics.
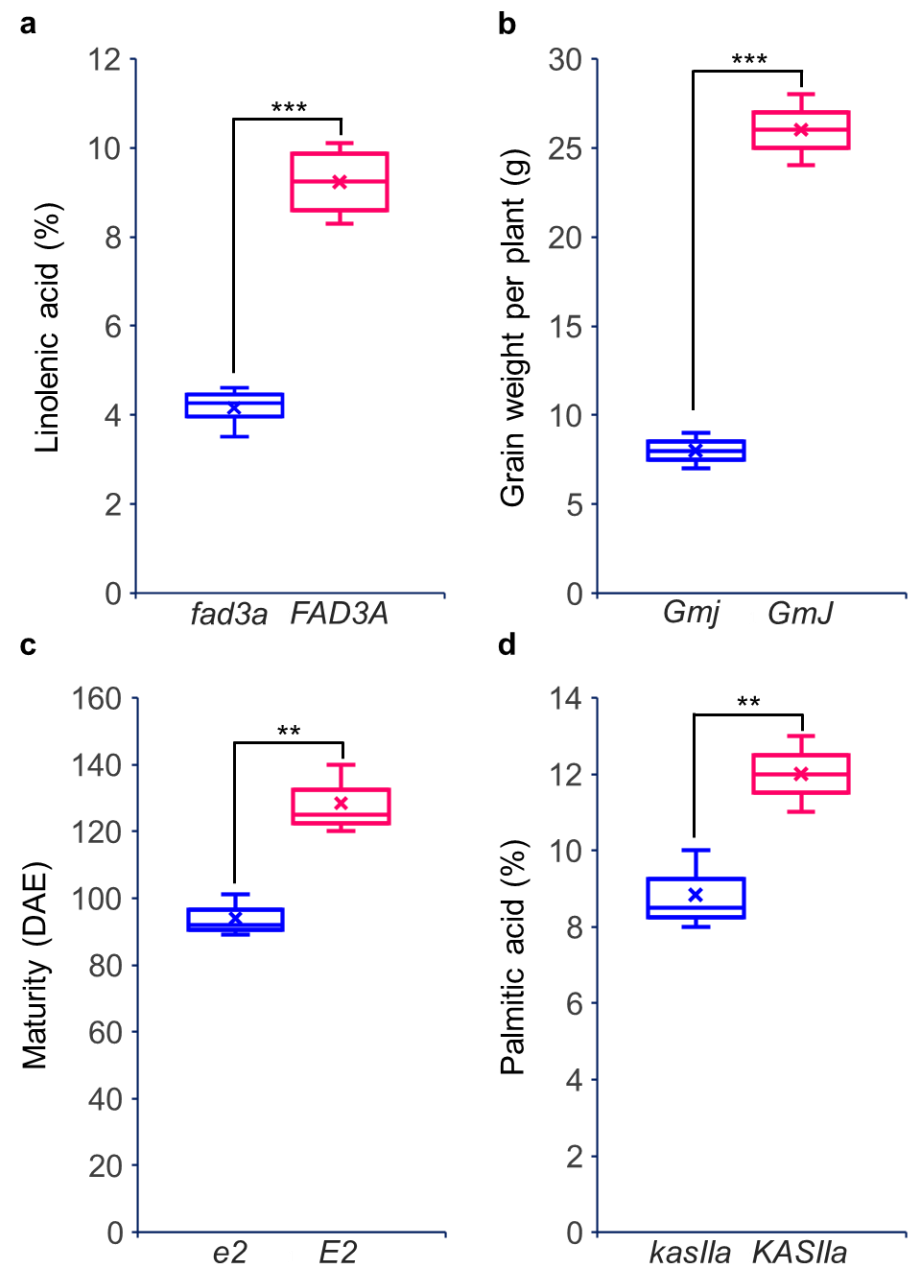
1 Figure 4. Phenotypic variation observed between accessions with (blue) and without (red) a

2 predicted LOF mutation in four different genes. (a) $F A D 3 A$, a key gene for linolenic acid synthesis;

3 (b) GmJ, a key gene of Long Juvenile trait; (c) GmGIa, a key gene controlling maturity; (d),

$4 \quad$ KASIIa, a key gene in the oil biosynthesis pathway.

\section{Discussion}

8 Using whole-genome sequencing data from a large collection of 1,007 soybean accessions, we

9 developed the first haplotype map of soybean (GmHapMap), a valuable resource for soybean genetic studies and breeding. A first challenge was to create a uniform and accurate catalogue of

11 nucleotide variation using a common version of the reference genome and a single bioinformatics

12 pipeline (Lek et al. 2016). The GmHapMap produced here is not only uniform but also it achieved

13 higher levels of genotype accuracy (>98\%) compared to previous studies (92-97\%) (Hwang et al.

14 2015). To create a representative haplotype map, a good level of saturation of both variants and

15 haplotypes is required. Close to $15 \mathrm{M}$ sequence variants (SNVs, MNVs, and Indels) were called

16 that captured nearly all polymorphisms with MAF > 1\% in the USDA $G$. max germplasm

17 collection (Song et al. 2013). The number of sequence variants did not increase significantly

18 beyond the first 600 accessions, suggesting that a collection of this size has succeeded in capturing

19 a sizeable fraction of worldwide nucleotide variation within cultivated soybean. Similarly, the

20 number of unique haplotypes (4.3M tag SNPs) also plateaued relatively early within this collection

21 of soybean germplasm. Together, these data suggest that the $15 \mathrm{M}$ variants captured in GmHapMap

22 are both highly accurate and comprehensive of the genetic diversity within cultivated soybean at

23 a worldwide level. 
1 GmHapMap brings more resolution to the within-species diversity of G. max. A lower level of

2 genomewide genetic diversity was observed here in soybean (mean $\theta_{\pi}=1.36 \times 10^{-3}$ ) compared to

3 other major crops such as rice $\left(\theta_{\pi}=2.29 \times 10^{-3}\right)\left(\right.$ Caicedo et al. 2007) and corn $\left(\theta_{\pi}=6.6 \times 10^{-3}\right)($

4 Gore et al. 2009). It is presumed that several genetic bottlenecks, as well as strong selection

5 pressure have reduced genetic diversity in soybean (Hyten et al. 2006). In addition, modern

6 soybean breeding is founded on a very limited number of the founder accessions (Hymowitz et al.

7 1983). We also noticed an average Nonsyn/Syn ratio of 1.49, which is higher than that reported in

8 other plants (sorghum (1.0), rice (1.2) and Arabidopsis (0.83) (Clark et al. 2007; McNally et al.

9 2009; Wang et al. 2015)). The greater accumulation of deleterious mutations in the soybean

10 genome could be attributed to (1) a reduced effective population size (Makino et al. 2018); (2) a

11 higher level of LD and the resulting 'hitchhiking' effect (Stephan et al. 2008); and (3) the

12 domestication-associated Hill-Robertson effect (Lu et al. 2006).

13 The GmHapMap was used as a reference panel and more than $21 \mathrm{~K}$ accessions that had been

14 previously genotyped using common approaches (SNP array and/or GBS) and obtained an

15 imputation accuracy of $92-96 \%$ for common variants and $\sim 80 \%$ for rare variants. The accuracy

16 levels, obtained here, are comparable to the $98 \%$ reported by Bukowski et al. (2018) in maize

17 (Bukowski et al. 2018). The success of untyped-genotype imputation depends critically on how

18 well a reference panel has captured the relevant haplotype diversity, as well as the marker density

19 of the experimental dataset (Browning \& Browning 2016). Here we document that GmHapMap

20 provides an extensive capture of SNP and haplotype diversity within cultivated soybeans

21 worldwide. It is likely that the lower imputation accuracy observed for the SNP array dataset can

22 be attributed to the relatively low marker density of this dataset. 
1 Enhanced datasets resulting from large-scale imputation can improve the efficacy of GWAS

2 analysis (Hao et al. 2009; Marchini \& Howie 2010). To illustrate the benefits of the GmHapMap

3 resource for GWAS, we performed an association analysis on soybean seed oil content using

4 imputed SNPs. A strong association with an imputed SNP residing in the NPC1 gene was detected

5 and its orthologue in Arabidopsis is known to contribute to seed oil content (Feldman et al. 2015).

6 Several studies in human (Li et al. 2009), cattle (Santana et al. 2014), pig (Yan et al. 2017), maize

7 (Yang et al. 2014) and rice (Wang et al. 2018) have demonstrated the capacity of imputation to

8 improve the power of GWAS analysis. In the coming years, we expect that soybean researchers

9 will deploy GmHapMap for imputation and more precise dissection of the genetic basis of complex

10 traits in soybean.

11 This is the first time that a comprehensive description of GCHs, for the complete set of genes

12 (55,589), has been achieved for a species. This catalogue of GCHs was obtained using

13 HaplotypeMiner (Tardivel et al. unpublished). Tardivel et al. reported that HaplotypeMiner

14 allowed the identification of SNP haplotypes for which $97.3 \%$ of lines sharing a same haplotype

15 were correctly identified as having the same allele (Tardivel et al. unpublished). It has been well

16 documented that haplotypes are more informative than single biallelic SNPs (Stephens et al. 2001).

17 Knowledge of the GCHs (and possibly alleles) can greatly facilitate the establishment of a

18 functional link between the various alleles of a gene and the associated phenotype. Haplotype-

19 phenotype association revealed the functional alleles of several genes in wheat (Jiang et al. 2015),

20 maize (Yang et al. 2013), rice (Si et al. 2016) and soybean (Langewisch et al. 2014). Knowledge

21 of the alleles present at one or many genes can be tremendously important to breeders. Epistatic

22 interactions between specific alleles as well as the effects of alleles at neighboring loci (carried 
1 along via linkage drag) can be very important when considering which combinations of alleles will

2 be most desirable to achieve a given phenotype.

3 A final aspect of this work is that the identification of LOF mutations in soybean protein-coding

4 genes. GmHapMap includes a set of nearly $11 \mathrm{~K}$ knocked-out genes. We recognized that this

5 catalogue of knocked-out genes is highly advantageous for soybean functional genomics for

6 investigation of gene function, and application as genetic makers in soybean breeding programs.

7 The next challenge will be to link genetic variation, GCHs, and LOFs derived from GmHapMap

8 with agronomic traits. This will need an extensive effort to measure phenotypes under multiple

9 field and laboratory conditions. We believe that GmHapMap will lead and accelerate the soybean

10 breeding efforts and future sustainable agriculture. 


\section{Methods}

\section{GmHapMap sequencing data}

3 Two collections of soybeans were used: a first set of 727 accessions for which whole-genome

4 sequencing had been previously released (Zhou et al. 2015; Maldonado dos Santos et al. 2016;

5 Valliyodan et al.2016; Fang et al. 2017; Song et al. 2017; Torkamaneh et al. 2017) and a second

6 set of 280 accession which were sequenced in this study. These were chosen to provide a more

7 balanced representation of various soybean growing areas in the world. Seeds were planted in

8 individual two-inch pots containing a single Jiffy peat pellet (Gérard Bourbeau \& fils inc. Quebec,

9 Canada). First trifoliate leaves from 12-day-old plants were harvested and immediately frozen in

10 liquid nitrogen. Frozen leaf tissue was ground using a Qiagen TissueLyser. DNA was extracted

11 from approximately $100 \mathrm{mg}$ of ground tissue using the Qiagen Plant DNeasy Mini Kit according

12 to the manufacturer's protocol. DNA was quantified on a NanoDrop spectrophotometer. Illumina

13 Paired-End libraries were constructed for 280 accessions using the KAPA Hyper Prep Kit (Kapa

14 Biosystems, Wilmington, Massachusetts, USA) following the manufacturer's instructions

15 (KR0961 - v5.16). Samples were sequenced on an Illumina HiSeq X10 platform at the McGill

University-Génome Québec Innovation Center in Montreal, QC, Canada.

\section{Nucleotide variants identification}

19 Sequencing reads from all 1,007 accessions were processed using the same analytical

21 genetic variants. In brief, the 100-150-bp paired-end reads were mapped against the G. max

22 reference genome [Gmax_275 (Wm82.a2)] (Schmutz et al. 2010). Then we removed variants if: 
1 1) they had more than two alleles, 2) an allele was not supported by reads on both strands, 3) the

2 overall quality (QUAL) score was <32, 4) the mapping quality (MQ) score was <30, 5) read depth

3 (minNR) was $<2$ and 6) the minor allele frequency (MinMAF) was $<0.0009$.

The SoySNP50K iSelect BeadChip has been used to genotype the entire USDA soybean

7 germplasm collection (Song et al. 2013). The complete dataset for 20,087 G. max and G. soja accessions genotyped with 42,508 SNPs was downloaded from Soybase (Grant et al. 2010). Of

9 these accessions, we randomly selected 50 accessions which were in common with the

10 GmHapMap collection. For these 50 accessions, we extracted their genotype calls at all SNP loci

11 for which data were available. This large set of SoySNP50K genotype calls $(2,125,400$ genotypes

12 or data points) was directly compared with the WGS-derived SNP calls (obtained using the Fast-

13 WGS pipeline) to assess genotype accuracy.

The functional impact of nucleotide variants was performed using the soybean genome using

17 SnpEff and SnpSift (Cingolani et al. 2012). Based on the genome annotation, nucleotide variants

18 were categorized on the basis of their location (exonic, intronic, splice sites, UTR ( $3 \& 5$ prime),

19 upstream and downstream regions (within $5 \mathrm{~kb}$ of a gene), and intergenic) and their predicted

20 functional impact (missense, nonsense, and silent). To determine LOF mutation, a database was

21 built using 55K soybean protein-coding genes (Gmax_275_Wm82.a2.v1.gene.gff3, from

22 Phytozome on Jan. 2016) for SnpEff. The LOF variants were extracted from the SnpEff-annotated 
1 VCF file using grep command lines. Variants were mapped on to transcripts annotated as

2 "protein_coding" and containing an annotated "START" codon, and then classified as

3 synonymous, missense, nonsense (stop codon-introducing, start/stop codon-disrupting or splice

4 site-disrupting (canonical splice sites)). In this work, we excluded transcripts labelled as NMD

5 (predicted to be subject to nonsense-mediated mRNA decay). We also applied another filtering

6 step, based on annotation, to identify high-confidence knocked-out genes. The genes with LOF

7 mutations were removed if (i) the 'REF' field in the input VCF file did not match the reference

8 genome, (ii) they had an incomplete transcript, or (iii) they did not have a proper START codon.

10 Population structure and genetic diversity

\section{Structure}

12 Population structure was estimated using a variational Bayesian inference implemented in

13 fastSTRUCTURE (Raj et al. 2014). Five runs were performed for each number of populations (K)

14 set from 1 to 15 using genomewide SNP data. The most likely K value was determined by the log

15 probability of the data $(\mathrm{LnP}(\mathrm{D}))$ and delta $\mathrm{K}$, based on the rate of change in $\mathrm{LnP}(\mathrm{D})$ between

16 successive K values. Similar analyses were performed separately for all 20 chromosomes of

17 soybean.

18 Genetic relationship

19 The evolutionary history was inferred using the Neighbor-Joining method (Saitou \& Nei 1987)

20 (rooted and unrooted) with the 12M genomewide SNPs identified in this study. The taxa were

21 clustered together using bootstrap test (1,000 replicates) (Felsenstein 1985). The tree was drawn

22 to scale, with branch lengths (next to the branches) in the same units as those of the evolutionary 
1 distances used to infer the phylogenetic tree. The evolutionary distances were computed using the

2 Maximum Composite Likelihood method (Tamura et al. 2004) and the units correspond to the

3 number of base substitutions per site. Evolutionary analyses were conducted in MEGA7 (Kumar

4 et al. 2016).

5 Genetic diversity

6 We measured the nucleotide diversity $(\pi)$ in sliding windows of $1000 \mathrm{bp}$ across the genome using

$7 \quad$ VCFtools (Danecek et al. 2011). The average pairwise divergence within a subpopulation $(\theta \pi)$ was

8 estimated for the whole genome among different subpopulations. Sliding windows of different

9 sizes $(1 \mathrm{~kb}, 7 \mathrm{~kb}, 10 \mathrm{~kb}$ and $100 \mathrm{~kb})$ that had a $90 \%$ overlap between adjacent windows were used

10 to estimate $\theta \pi$ for both whole genome and each chromosome. To display the pattern in each

11 chromosome, a window of $100 \mathrm{~kb}$ was used.

14 Genomewide pairwise linkage disequilibrium (LD) analysis ( $\mathrm{r}^{2}$ and $\mathrm{D}^{\prime}$ ) was performed using all

15 nucleotide variants from the GmHapMap dataset. The average $r^{2}$ value was calculated for each

16 length of distance (<1000 bp), and LD decay calculated using PopLDdecay (Zhang et al. 2018).

17 For tag SNP selection, we used PLINK (Purcell et al. 2007) to calculate LD between each pair of

18 SNPs within a sliding window of 50 SNPs and we removed all but one SNP that were in perfect

$19 \mathrm{LD}(\mathrm{LD}=1)$; the remaining SNPs were deemed tag SNPs. 


\section{Phasing, identification of identity by descent (IBD) and imputation}

2 The IBD analysis was conducted using BEAGLE v4.1 (Browning \& Browning 2016). In brief, to

3 identify IBD segments, the genotypic dataset was phased using BEAGLE with 50 iterations for

4 each chromosome. The output of these calculations was a series of "putative" IBD segments shared

5 between pairs of individuals. Each segment comes with the following information attached: IDs

6 for the pair of individuals, start and end position of the IBD segment, and probability score (LOD

7 score). We filtered these segments using LOD score and the length of IBD.

\section{$9 \quad$ Imputation of untyped variants}

10 We used two reference panels for untyped-variant imputation. The 'REF-I' panel includes 1,006 11 accessions from GmHapMap with the entire SNP dataset, while the 'REF-II' panel includes 1,006

12 accessions and only 1.9M tag SNPs from genic regions (tag SNPs in genic regions or within $2 \mathrm{~kb}$

13 of a gene). These two reference panels were created for all 20 chromosomes of soybean and were

14 phased using BEAGLE v4.1 (Browning \& Browning 2016) with 100 iterations.

15 As initial lower density datasets, we used three collections of soybean accessions genotyped with commonly used genotyping tools. A first set of 20,087 accessions (the entire USDA Soybean

17 Germplasm Collection) had been characterized using the SoySNP50K iSelect Bead Chip (Song et al. 2013) to yield a set of $43 \mathrm{~K}$ polymorphic markers. A second set comprised 1,531 accessions which had been subjected to genotyping-by-sequencing (GBS; ApeKI protocol) (Sonah et al. 2013)

20 and in which SNPs had been called using the Fast-GBS pipeline (Torkamaneh et al. 2017). Finally,

21 a third set of 1,531 accessions (GBS set) with a combined SNP catalogue derived from GBS and

\section{SoySNP50K (Supplementary Note).}


1 Phasing and imputation were performed using BEAGLE v4.1 (Browning \& Browning 2016) for

2 each chromosome with the following parameters: (i) nthreads = 10 (number of threads); (ii)

3 window $=100,000$ (number of markers in a sliding window); (iii) overlap $=50,000$ (number of

4 overlapping markers between adjacent windows); (iv) niterations $=100$ (number of phasing

5 iterations) and (v) err $=0.00001$ (the allele miscall rate).

6

7

\section{Determining the imputation accuracy}

The WGS SNP data from 1,006 of the 1,007 resequenced accessions were used as a reference panel to impute untyped variants. The remaining line was kept out of the reference panel to determine how accurately data at untyped loci (present in the GmHapMap data but absent from the low-density genotype catalogue) could be imputed in this accession. We performed three such permutations where a single accession was kept aside to estimate imputation accuracy. For these lines purposely excluded from the reference panel, we compared the imputed genotypes against the genotypes called at these same loci following WGS.

\section{Genomewide association analysis}

Sonah et al. (2013) described a set of QTLs using GWA analysis on a subset of 139 soybean accessions. These accessions were genotyped via GBS. We imputed untyped variants on this lowdensity genotype dataset from GmHapMap in $1 \mathrm{Mb}$ of chromosome 14 , encompassing a QTL for seed oil content. GWA analysis was conducted using GAPIT R package (Lipka et al. 2012) using a MLMM model (Segura et al. 2012). A candidate gene was identified using SoyBase database 
1 (Grant et al. 2010) and The Arabidopsis Information Resource (TAIR)

2 [https://www.arabidopsis.org/servlets/TairObject?type=gene\&name=AT4G38350.1]

$4 \quad$ Identification of gene-centric haplotypes

5 The identification of GCHs was performed using the HaplotypeMiner $\mathrm{R}$ package

6 (https://github.com/malemay/HaplotypeMiner) with the entire SNP dataset on 55,381 protein-

7 coding genes in the soybean genome. In brief, the following parameters were used: (i) R2_measure

$8=$ "r2s" (the estimation of linkage disequilibrium between markers was measured based on

9 corrected $r_{v s}^{2}$ which takes into account information related to genetic relatedness and population

10 structure); (ii) cluster_R2 = "r2s" (LD measure to use in the clustering step); (iii)

11 max_missing_threshold $=0.05$ (the maximum proportion of missing genotypes allowed for a

12 marker); (iv) max_het_threshold $=0.01$ (the maximum proportion of heterozygous genotypes

13 allowed for a marker); (v) min_allele_count $=4$ (the minimum number of times the minor allele

14 has to be seen for a marker to be retained); (vi) cluster_threshold $=0.9$ (the minimum LD beyond

15 which markers were clustered); (vii) max_flanking_pair_distance $=10000$ (the maximum distance

16 (in bp) that can separate two markers in LD at the final selection step: (viii)

17 max_marker_to_gene_distance $=6000$ (the maximum distance (in bp) from a marker to the center

18 of the gene of interest); (ix) marker_independence_threshold $=0.8$ (the minimum LD for two

19 markers to be considered in LD at the final selection step). 
2 We detected putative duplicated genes, presumably derived from WGD or gene duplication, using

3 protein homology analysis integrated in the Phytozome (Goodstein et al. 2012) and SoyBase

4 (Grant et al. 2010) databases. Protein homologs were identified using dual-affine Smith-Waterman

5 alignments between the predicted translation product of the selected transcript (aka query gene)

6 and all other predicted proteins in the soybean genome. We identified duplicated genes with $90 \%$

7 identity (ID $\geq 90), 90 \%$ coverage $(C V \geq 90)$, and $5 \%$ size difference $(\mathrm{SD} \leq 5)$ threshold.

\section{Code availability}

10 The bioinformatics codes and scripts applied in this study for variant calling, population structure

11 analysis, genetic diversity, tag SNP selection, imputation, GWAS, annotation and GCHs detection

12 are publicly available at https://figshare.com/account/home\#/projects/56921.

\section{Data availability}

15 The datasets produced in this study (GmHapMap nucleotide variants (complete dataset), reference panels (REF-I and REF-II), annotated GmHapMap nucleotide variants, GCHs for all 55K genes

17 and LOF variants and genotypes) are publicly available at

18 https://figshare.com/account/home\#/projects/56921 


\section{Acknowledgments}

2 This work was supported by the SoyaGen grant (www.soyagen.ca) awarded to F. Belzile and

3 funded by Génome Québec, Genome Canada, the government of Canada, the Ministère de

4 l’Économie, Science et Innovation du Québec, Semences Prograin Inc., Syngenta Canada Inc.,

5 Sevita Genetics, Coop Fédérée, Grain Farmers of Ontario, Saskatchewan Pulse Growers, Manitoba

6 Pulse \& Soybean Growers, the Canadian Field Crop Research Alliance and Producteurs de grains

7 du Québec. The work conducted by the US DOE Joint Genome Institute is supported by the Office

8 of Science of the US DOE under Contract DE-AC02-05CH11231. We thank Dr. Brain Boyle to

9 help for DNA library construction. We thank the Joint Genome Institute and collaborators for pre-

10 publication access to the Glycine max W82 V2 genome sequence. We also thank Dr. Gary Stacy

11 and Dr. Shakhawat Hossain for pre-publication access to the soybean transcriptome atlas.

\section{Author contributions}

13 DT and FB conceived the project. DT and JL contributed to programming and analysis of genomic 14 data. DT carried out genetic diversity analysis, imputation, GCHs and identification of LOFs. BV

15 and $\mathrm{HN}$ provided sequence data for American accessions. RA provided data for Brazilian 16 accessions. DT, FB, EC, IR and LO provided data for Canadian accessions and also carried out

17 NIRS analysis. AS and JS carried out the identification of gene duplication. DT and FB contributed 18 to writing the manuscript.

20 The authors declare that they have no competing interests. 


\section{References}

Browning BL, Browning SR. 2016. Genotype Imputation with Millions of Reference Samples. American Journal of Human Genetics. 98(1):116-126. doi:10.1016/j.ajhg.2015.11.020.

Bukowski R, Guo X, Lu Y, et al. 2018. Construction of the third-generation Zea mays haplotype map. GigaScience. 7(4):gix134. doi:10.1093/gigascience/gix 134.

Caicedo, A.L. et al. 2007. Genome-wide patterns of nucleotide polymorphism in domesticated rice. PLoS Genet. 3, 1745-1756.

Cingolani, P., Platts, A., Wang, L.L., et al. 2012. A program for annotating and predicting the effects of single nucleotide polymorphisms, SnpEff: SNPs in the genome of Drosophila melanogaster strain w1118; iso-2; iso-3. Fly. 6(2):80-92. doi:10.4161/fly.19695.

Clark, R.M. et al. 2007. Common sequence polymorphisms shaping genetic diversity in Arabidopsis thaliana. Science 317, 338-342.

Danecek P, Auton A, Abecasis G, Albers CA, Banks E, et al. 2011. The Variant Call Format and VCFtools. Bioinformatics. doi:10.1093/bioinformatics/btr330.

Djanaguiraman M et al. 2018. Reproductive success of soybean (Glycine max L. Merril) cultivars and exotic lines under high daytime temperature. Plant, Cell \& Environment. https://doi.org/10.1111/pce.13421.

Fang C, Ma Y, Wu S, et al. 2017. Genome-wide association studies dissect the genetic networks underlying agronomical traits in soybean. Genome Biology. 18:161. doi:10.1186/s13059-0171289-9.

Feldman MJ, Poirier BC, Lange BM. 2015. Misexpression of the Niemann-Pick disease type C1 (NPC1)-like protein in Arabidopsis causes sphingolipid accumulation and reproductive defects. Planta. 242: 921. https://doi.org/10.1007/s00425-015-2322-4

Felsenstein J. 1985. Confidence limits on phylogenies: an approach using the bootstrap. Evolution. 1985 Jul;39(4):783-791. doi: 10.1111/j.1558-5646.1985.tb00420.x.

Goettel W, Ramirez M, Upchurch RG, An YC. 2016. Identification and characterization of large DNA deletions affecting oil quality traits in soybean seeds through transcriptome sequencing analysis. Theoretical and Applied Genetics. 129:1577-1593. doi:10.1007/s00122-016-2725-z.

Goodstein, D.M., Shu, S., Howson, R., et al. 2012. Phytozome: a comparative platform for green plant genomics. Nucleic Acids Research. 40(Database issue):D1178-D1186. doi:10.1093/nar/gkr944.

Gore, M.A. et al. 2009. A first-generation haplotype map of maize. Science 326, 1115-1117.

Grant, D., Nelson, R.T., Cannon, S.B., and Shoemaker, R.C. 2010. SoyBase, the USDA-ARS soybean genetics and genomics database. Nucl. Acids Res. D843-D846. doi: 10.1093/nar/gkp798 
Hao K, Chudin E, McElwee J, Schadt E. 2009. Accuracy of genome-wide imputation of untyped markers and impacts on statistical power for association studies. BMC Genet. 10:27. doi:10.1186/14712156-10-27PMID:19531258

Hwang, S., Kim, E., Lee, I. and Marcotte, E.M. 2015. Systematic comparison of variant calling pipelines using gold standard personal exome variants. Scientific Reports. 17875. 6 doi:10.1038/srep17875

Hymowitz, T. \& Harlan, J.R. 1983. Introduction of soybean to North America by Samuel Bowen in 1765. Econ. Bot. 37, 371-379. Hymowitz, T. 1970. On the domestication of soybean. Econ. Bot. 24, 408-421.

Hyten, D.L. et al. 2006. Impacts of genetic bottlenecks on soybean genome diversity. Proc. Natl. Acad. Sci. USA 103, 16666-16671.

Jiang Y., Jiang Q., Hao C., Hou J., Wang L., Zhang H., et al. 2015. A yield-associated gene TaCWI, in wheat: its function, selection and evolution in global breeding revealed by haplotype analysis. Theor. Appl. Genet. 128 131-143. 10.1007/s00122-014-2417-5

Kumar S, Stecher G, Tamura K. 2016. MEGA7: Molecular Evolutionary Genetics Analysis Version 7.0 for Bigger Datasets. Mol Biol Evol. 33(7):1870-4. doi: 10.1093/molbev/msw054.

Langewisch T, Zhang H, Vincent R, Joshi T, Xu D, Bilyeu K. 2014. Major Soybean Maturity Gene Haplotypes Revealed by SNPViz Analysis of 72 Sequenced Soybean Genomes. PLoS ONE 9(4): e94150.

Lek, M. et al. 2016. Analysis of protein-coding genetic variation in 60,706 humans. Nature 536, 285-291.

Li Y, Willer C, Sanna S. 2009. Genotype Imputation. Annu. Rev. Genomics Hum. Genet. 10:387406. doi:10.1146/annurev.genom.9.081307.164242

Lipka, A.E., Tian, F., Wang, Q., Peiffer, J., Li, M., Bradbury, P.J., et al. 2012. GAPIT: genome association and prediction integrated tool. Bioinformatics. 28 (18): 2397-2399. doi: 10.1093/bioinformatics/bts444

Lu S et al. 2017. Natural variation at the soybean J locus improves adaptation to the tropics and enhances yield. Nature Genetics. 49, 773-779.

Lu, J. et al. 2006. The accumulation of deleterious mutations in rice genomes: a hypothesis on the cost of domestication. TIG 22, 126-131.

MacArthur DG et al. 2012. A systematic survey of loss-of-function variants in human proteincoding genes. Science 335, 823-828. 
Maldonado dos Santos JV et al. 2016. Evaluation of genetic variation among Brazilian soybean cultivars through genome resequencing. BMC Genomics 17:110.

Maldonado dos Santos JV et al. 2016. Evaluation of genetic variation among Brazilian soybean

4 cultivars through genome resequencing. BMC Genomics 17:110.

Marchini J, Howie B. 2010. Genotype imputation for genome-wide association studies. Nature

6 Reviews Genetics volume 11, pages 499-511.

McNally, K.L. et al. 2009. Genome wide SNP variation reveals relationships among landraces and modern varieties of rice. Proc. Natl. Acad. Sci. USA 106, 12273-12278.

9 Purcell, S., Neale, B., Todd-Brown, K., Thomas, L., Ferreira, M.A., Bender, D., et al. 2007. PLINK: a tool set for whole genome association and population-based linkage analyses. Am. J. Hum. Genet. 81(3):559-75

Qiu, L.J. et al. 2013. A platform for soybean molecular breeding: the utilization of core collections for food security. Plant Mol. Biol. 83, 41-50.

Raj, A., Stephens, M., and Pritchard, J.K. 2014. fastSTRUCTURE: Variational Inference of Population Structure in Large SNP Data Sets. Genetics. 197:573-589

Ray DK, Mueller ND, West PC, Foley JA. 2013. Yield Trends Are Insufficient to Double Global Crop Production by 2050. PLoS ONE 8(6): e66428.doi:10.1371/journal.pone.0066428

Reinprecht Y, and Pauls KP. 2016. Microsomal Omega-3 Fatty Acid Desaturase Genes in Low Genetics. 7: 38.

Roulin, A. et al. 2013. The fate of duplicated genes in a polyploid plant genome. Plant J 73, 143153.

Saitou N and Nei M. 1987. The neighbor-joining method: a new method for reconstructing phylogenetic trees. Molecular Biology and Evolution. 4 (4): 406

Santana MH, Utsunomiya YT, Neves HH, Gomes RC, Garcia JF, Fukumasu H,et al. 2014. Genome-wide association analysis of feed intake and residual feed intake in Nellore cattle. BMCGenet. 15:21. doi:10.1186/1471-2156-15-21 the palaeopolyploid soybean. Nature. 463(7278):178-183. Genet 44: 825-830. 
Sonah H., O'Donoughue L., Cober E., Rajcan I., Belzile F. 2015. Identification of loci governing eight agronomic traits using a GBS-GWAS approach and validation by QTL mapping in soya bean. Plant Biotech. J. 3, 10. 10.1111/pbi.12249

Sonah, H., Bastien, M., Iquira, E., Tardivel, A., Legare, G., Boyle, B., et al. 2013. An Improved Genotyping by Sequencing (GBS) Approach Offering Increased Versatility and Efficiency of SNP Discovery and Genotyping. PLoS ONE. 8(1): e54603. doi: 10.1371/journal.pone.0054603 PMID: 23372741

Song Q et al. 2017. Genetic Characterization of the Soybean Nested Association Mapping Population. Plant Genome. 2017 Jul;10(2). doi: 10.3835/plantgenome2016.10.0109.

Song Q, Hyten DL, Jia G, et al. 2013. Development and Evaluation of SoySNP50K, a HighDensity Genotyping Array for Soybean. Zhang T, ed. PLoS ONE. 8(1):e54985. doi:10.1371/journal.pone.0054985.

Stephan W, Song YS, Langley CH. 2006. The Hitchhiking Effect on Linkage Disequilibrium Between Linked Neutral Loci. Genetics. 172(4):2647-2663. doi:10.1534/genetics.105.050179.

Stephens J. C., Schneider J. A., Tanguay D. A., Choi J., Acharya T., Stanley S. E., et al. 2001. Haplotype variation and linkage disequilibrium in 313 human genes. Science $293489-493$. $10.1126 /$ science. 1059431

Tamura K, Nei M, Kumar S. 2004. Prospects for inferring very large phylogenies by using the neighbor-joining method. Proc Natl Acad Sci U S A. 101:11030-11035.

Tardivel et al. (Unpublished) A systematic gene-centric approach to define haplotypes and identify alleles based on dense SNP datasets.

Torkamaneh D, Laroche J, Rajcan I, Belzile F. 2018. Identification of candidate domesticationrelated genes with a systematic survey of loss-of-function mutations. The Plant Journal. doi: 10.1111/tpj.14104.

Torkamaneh D, Laroche J, Tardivel A, O’Donoughue L, Cober E, Rajcan I, Belzile F. 2017. Comprehensive Description of Genome-Wide Nucleotide and Structural Variation in ShortSeason Soybean. Plant Biotechnology Journal.

Torkamaneh, D., Laroche, J., Bastien, M., Abed, A., Belzile, F. 2017. Fast-GBS: a new pipeline for the efficient and highly accurate calling of SNPs from genotyping-by-sequencing data. BMC Bioinformatics. doi: 10.1186/s12859-016-1431-9 M. Ishimoto, K. Harada. 2014. Natural variation in the genes responsible for maturity loci E1, E2, E3 and E4 in soybean. Ann. Bot. 113: 429-441.

34 Valliyodan, B., Qiu, D., Patil, G. et al. 2016. Landscape of genomic diversity and trait discovery 
1 Wang Y, Tan L, Fu Y, Zhu Z, Liu F, Sun C, et al. 2015 Molecular Evolution of the Sorghum

2 Maturity Gene Ma3. PLoS ONE 10(5): e0124435.

3 Wang, Diane R. et al. 2018. An Imputation Platform to Enhance Integration of Rice Genetic

4 Resources. Nature Communications. 9: 3519. PMC. Web. 6 Oct. 2018.

5 Watanabe S, Harada K, Abe J. 2012. Genetic and molecular bases of photoperiod responses of

6 flowering in soybean. Breeding Science. 61(5):531-543. doi:10.1270/jsbbs.61.531.

7 Yan, G., Qiao, R., Zhang, F., Xin, W., Xiao, S., Huang, T., ... Huang, L. 2017. Imputation-Based

8 Whole-Genome Sequence Association Study Rediscovered the Missing QTL for Lumbar Number

9 in Sutai Pigs. Scientific Reports, 7, 615.

10 Yang N, Lu Y, Yang X, Huang J, Zhou Y, Ali F, et al. 2014. Genome Wide Association Studies

11 Using a New Nonparametric Model Reveal the Genetic Architecture of 17 Agronomic Traits in an

12 Enlarged Maize Association Panel. PLoS Genet 10(9): e1004573.

13 Yang Q., Li Z., Li W., Ku L., Wang C., Ye J., et al. 2013. CACTA-like transposable element in

14 ZmCCT attenuated photoperiod sensitivity and accelerated the postdomestication spread of maize.

15 Proc. Natl. Acad. Sci. U.S.A. 110 16969-16974. 10.1073/pnas.1310949110

16 Zhang $\mathrm{C}$ et al. 2018. PopLDdecay: a fast and effective tool for linkage disequilibrium decay

17 analysis based on variant call format files. Bioinformatics. doi: 10.1093/bioinformatics/bty875.

18 Zhou Z, Jiang Y, Wang Z, Gou Z, Lyu J et al. 2015. Resequencing 302 wild and cultivated

19 accessions identifies genes related to domestication and improvement in soybean. Nature

20 Biotechnology. 33, 408-414. doi:10.1038/nbt.3096. 